\title{
Vagal Withdrawal and Sympathetic Overactivity Contribute to the Genesis of Early-Onset Pregnancy-Induced Hypertension
}

\author{
G. K. Pal, ${ }^{1}$ P. Shyma, ${ }^{1}$ S. Habeebullah, ${ }^{2}$ Pravati Pal, ${ }^{1}$ Nivedita Nanda, ${ }^{3,4}$ and P. Shyjus ${ }^{2}$ \\ ${ }^{1}$ Department of Physiology, Jawaharlal Institute of Postgraduate Medical Education and Research (JIPMER), \\ Puducherry 605 006, India \\ ${ }^{2}$ Department of Obstetics and Gynecology, Jawaharlal Institute of Postgraduate Medical Education and Research (JIPMER), \\ Puducherry 605 006, India \\ ${ }^{3}$ Department of Biochemistry, Jawaharlal Institute of Postgraduate Medical Education and Research (JIPMER), \\ Puducherry 605 006, India \\ ${ }^{4}$ Pondicherry Institute of Medical Sciences (PIMS), Pondicherry 605 014, India
}

Correspondence should be addressed to G. K. Pal, gopalpravati@sify.com

Received 4 November 2010; Revised 31 December 2010; Accepted 10 February 2011

Academic Editor: Markus Schlaich

Copyright ( 2011 G. K. Pal et al. This is an open access article distributed under the Creative Commons Attribution License, which permits unrestricted use, distribution, and reproduction in any medium, provided the original work is properly cited.

Objective. In this study, we have assessed sympathovagal imbalance (SVI) by spectral analysis of heart rate variability (HRV) that contributes to the genesis of early-onset PIH. Methods. Body mass index (BMI), basal heart rate (BHR), blood pressure (BP) and HRV indices such as LFnu, HFnu, LF-HF ratio, mean RR, SDNN and RMSSD were assessed in normal pregnant women (Control group) and pregnant women having risk factors for PIH (Study group) at all the trimesters pregnancy. Retrospectively, those who did not develop PIH (Study group I) were separated from those who developed PIH (Study group II). Study group II was subdivided into early-onset and late-onset PIH. Sympathovagal balance (LF-HF ratio) was correlated with BMI, BHR and BP. Results. LF-HF ratio was significantly high in study group II compared to study group I and control group, and in early-onset PIH group compared to the late-onset category at all the trimesters of pregnancy, which was significantly correlated with BHR and BP. Alteration in HFnu in early-onset category was more prominent than the alteration in LFnu. Conclusion. Though the SVI in PIH is contributed by both sympathetic overactivity and vagal withdrawal, especially in early-onset type, SVI is mainly due to vagal inhibition.

\section{Introduction}

Hypertensive disorders of pregnancy contribute significantly to still births and neonatal and maternal morbidity and mortality, especially in developing countries of south-east Asia [1]. In India, pregnancy-induced hypertension (PIH) accounts for about 50,000 deaths a year [2]. The major cause of maternal and fetal deaths in PIH is due to its late diagnosis and inadequate treatment. The clinical and laboratory characteristics of hypertension associated with or induced by pregnancy are difficult to differentiate from those of hypertension independent of pregnancy [3]. Pregnancy may induce hypertension in women who are normotensive before pregnancy and may aggravate hypertension in those who are hypertensive before pregnancy [4]. As a result, usually precarious delay occurs in the initiation of appropriate treatment for PIH.
Though the etiology of PIH is not exactly known, it has been well established that the disease is characterized by low circulating volume and high vascular resistance $[5,6]$. Usually, two physiological factors that largely contribute to the genesis of hypertension are decreased size of the vascular compartment (vasoconstriction) and increase volume of the compartment (increased blood volume) [7]. However, in this dysfunction, hypertension develops inspite of low blood volume, which clearly indicates that the primary contributor to PIH is intense vasoconstriction induced by sympathetic overreactivity [6]. Recent reports from our laboratory confirmed that detection of subtle increase in sympathetic tone by spectral HRV analysis in the first trimester of pregnancy in women with high risks for PIH predicts the development of PIH in these women that clinically manifests in third trimester of pregnancy $[8,9]$. 
Though, PIH usually develops in the later part of third trimester of pregnancy, especially towards term, in some patients disease manifests early, before completion of 34th week of pregnancy (early-onset PIH), and often in them the disease progresses rapidly [4]. Therefore, unless repeated screening is performed at short intervals in this early-onset category, patient reports in the advanced stage of the disease that creates difficulty in the management of this dysfunction. To the best of our knowledge, no report is available yet to assess the nature of alteration in sympathovagal balance that leads to the causation of early-onset PIH. Therefore, in the present study we have performed spectral analysis of HRV to understand the pathophysiology of early-onset PIH that may help develop measures to prevent the occurrence of this common dysfunction.

\section{Methods}

The present study was conducted in the Department of Physiology, Jawaharlal Institute of Postgraduate Medical Education and Research (JIPMER), Pondicherry, India. After obtaining approval of the project plan from research and ethics committees of JIPMER, 234 subjects (50 in control group, 184 in study group) were recruited from the outpatient unit of the obstetrics and gynecology department of JIPMER. Written informed consent was obtained from all the participants prior to initiation of the study. Subjects of study group included pregnant women who had at least any one of the established risk factors for PIH [10] such as previous history of pre-eclampsia, extremes of reproductive age, first pregnancy, diabetes mellitus, pre-existing hypertension, and multiple pregnancy, Subjects of control group included pregnant ladies who had none of the above-mentioned risk factors for PIH.

Twelve subjects in the control group and seven subjects in the study group did not turn up for second and third trimester recordings. Therefore, these subjects were excluded from the study. Rest all subjects (38 in the control group and 177 in the study group) attended obstetrics OPD at all three trimesters of pregnancy and also reported to polygraph laboratory for recording of various parameters. All subjects were followed up till term and any incidence of PIH was recorded. Out of 177 study subjects, 31 developed PIH during their course of pregnancy and were included in the study group. Study group subjects were further divided into early-onset PIH (who developed PIH before completion of 34th week) and late-onset PIH (who developed PIH after completion of 34th week) based on the criteria of our hospital and others [4].

Subjects were advised to come to obstetrics OPD for three antenatal check-ups during their pregnancy and from OPD they were instructed to report to polygraph laboratory of physiology department for recording of various parameters during each check-up. Accordingly, three recordings were performed on all subjects at different gestational periods; the 1st recording at completion of 1st trimester (towards the end of 12th week), the 2nd recording at completion of 2 nd trimester (towards the end of the 24th week), and the 3rd recoding at the end of the 31st week (as previous records of Obstetrics and Gynecology Department of JIPMER indicated that PIH usually occurs between the 32 nd weeks and term). The subjects reported to polygraph laboratory about two hours after a light breakfast devoid of coffee or tea. Height and weight were measured to calculate body mass index (BMI). Blood pressure (BP) was recorded using Colin PressMate 8800 (Colin Corporation, Japan) noninvasive blood pressure monitor.

Following 10 minutes of supine rest in polygraph laboratory (room temp maintained at $25^{\circ} \mathrm{C}$ ), basal heart rate (BHR), systolic blood pressures (SBP), and diastolic blood pressure (DBP) were recorded. For recording of shortterm HRV, recommendation of the Task Force on HRV was followed [11]. For the purpose, ECG electrodes were connected and Lead II ECG was acquired at a rate of 1000 samples/second during supine rest using BIOPAC MP 100 data acquisition system (BIOPAC Inc., USA). The data was transferred from BIOPAC to a windows-based PC with Acqknowledge software version 3.8.2. Ectopics and artifacts were removed from the recorded ECG. RR tachogram was extracted from the edited 256 sec ECG using the R wave detector in the Acqknowledge software and saved in ASCII format which was later used offline for short-term HRV analysis. HRV analysis was done using the HRV analysis software version 1.1 (Biosignal Analysis group, Finland). Mean RR was measured in second(s). Variance, defined as power in a portion of the total spectrum of frequencies was measured in milliseconds squared $\left(\mathrm{ms}^{2}\right)$. Different frequency domain indices such as total power (TP), lowfrequency component expressed as normalized unit (LFnu), high-frequency component expressed as normalized unit (HFnu) and LF/HF ratio, and time domain indices such as mean RR, standard deviation of normal to normal intervals $(\mathrm{SDNN})$ and square root of the mean squared differences of successive normal to normal intervals (RMSSD) of HRV were calculated. All subjects were followed up till term and any incidence of PIH (DBP $90 \mathrm{mmHg}$ or above or SBP $140 \mathrm{mmHg}$ or above or both) was recorded $[3,4]$.

2.1. Statistical Analysis. The SPSS version 13 was used for statistical analysis. All the data were expressed as mean \pm SD. One-way ANOVA with Tukey-Kramer post hoc was used in analyzing data between the three groups and across the three different weeks within the groups. Student's unpaired $t$ test was used to detect the level of significance between earlyonset and late-onset PIH groups. The association between LF-HF ratio with BMI, BHR, and blood pressures was assessed by Pearson correlation analysis. The $P$-values less than .05 were considered significant.

\section{Results}

Two-hundred fifteen subjects (38 control subjects and 177 subjects having risk factor for $\mathrm{PIH}$ ) attended obstetrics OPD regularly and also reported to polygraph laboratory at all three trimesters of pregnancy for recording of various parameters. Out of 177 women having risk factors for $\mathrm{PIH}$, 
31 (17.51\%) developed PIH (Study group II). However, two subjects developed PIH before the 31st week of pregnancy and therefore, they do not have the 31st week recordings ( $n=29$ at the 31 st week). All other subjects developed PIH between the 32nd week and term. The 146 subjects who had risk factors for PIH, but did not develop PIH, were included in study group I.

3.1. General Parameters. There was no significant difference in age between the subjects of control group and study groups (Table 1). The body weight and BMI of subjects of study group II (those who developed PIH) was significantly more than that of the study group I (those who did not develop PIH) and control group at all the three gestational weeks of recordings. However, increase in body weight and BMI of subjects of control group and study group I, but not of the subjects of study group II was statistically significant at the 31 st week recording compared to their own 12th week recording.

The basal heart rate (BHR) of the subjects of study group II was significantly more at all the three trimesters compared to the subjects of control and study group I, though the general level of significance was reduced sequentially from the 12th week $(P<.0001)$ to the 31st week $(P=.0096)$ (Table 1). Yet, in control group and study group I, but not in study group II, the increase in BHR at the 31st week was highly significant compared to their own 12th and 24th week values.

The SBP study group II was significantly more than that of the study group I and control group $(P<.001)$ at all the three trimesters of pregnancy, whereas SBP of study group I was significantly more than that of the control group $(P<.01)$ only at 31 st week. The increase in SBP of study groups I and II, but not of the control group at the 31st week was significantly higher compared to their own 12th week recording (Table 1).

The DBP of study group II was significantly more than that of the study group I and control group at all the three trimesters of pregnancy. The increase in DBP of study group I and II, but not of the control group at the 31st week was significantly higher compared to their own 12th and 24th week recordings. Also, the DBP of study group II at the 24th week was significantly more $(P<.05)$ than its own 12 th week recording (Table 1).

3.2. Frequency Domain Indices of HRV. Total power (TP) of HRV spectrum was reduced significantly in study group II in comparison to study group I and control group at all the trimesters of pregnancy (Table 2), whereas reduction in TP in study group I compared to that of control group was significant only at the 31st week. However, the reduction in TP at the 31st week in study group I was statistically significant compared to its own 24th and 12th week recordings.

The LFnu of study group II was significantly higher compared to that of control group at all the three trimesters of pregnancy and to that of study group I at the 12th and 24th weeks of pregnancy (Table 2). However, the increase in
LFnu of control group and study group I at the 31st week, but not the study group II compared to their own 12th week recordings was statistically significant.

Though the HFnu was decreased in study group II compared to control group in all the three recordings, the decrease was significant $(P<.05)$ only at the 12th week (Table 2). Nevertheless, only in study group I the decrease in HFnu was statistically significant compared to its own 12th week recording.

The LF-HF ratio of study group II was significantly more compared to that of control group and study group I at all three trimester recordings (Table 2). Nonetheless, the increase in LF-HF ratio at the 31st week recording of control group and study group I, but not in study group II was significantly higher compared to their own 12th week and the 24th week recordings, in which the level of significance was more in study group I.

3.3. Time Domain Indices of HRV. The decrease in mean RR of study group II was significant compared to that of control group and study group I at the 12th and 24th week recordings (Table 2). Though the decrease in mean RR at the 31st week compared to their own 12th and 24th week values was significant in control group and study group I, the decrease was not significant for study group II.

The decrease in SDNN in study group II compared to that of control group and study group I was statistically significant $(P<.05)$ only at the 12 th week recording. RMSSD in study groups I and II was decreased significantly $(P<.05)$ compared to the control group only at the 31 st week. The decrease in RMSSD in study group II at the 31st week was significant $(P<.05)$ compared to its own 12th week value. Also, the decrease in RMSSD in study group I at the 31st week was significant compared to its own 12th week $(P<.001)$ and 24th week $(P<.05)$ values.

3.4. Early-Onset versus Late-Onset PIH. Out of $31 \mathrm{PIH}$ subjects, 15 developed PIH before completion of 34 weeks (those include two subjects who developed PIH before the 31st weeks), and they all were categorized as early-onset PIH, and 16 developed PIH after completion of 34 weeks and were classified as late-onset PIH.

3.4.1. General Parameters. There was no difference in age between subjects of early-onset and late-onset PIH groups (Table 3). There was no difference in body weight and BMI between subjects of early-onset and late-onset PIH groups at all the three trimester recordings (Table 3 ). Also, the increase in body weight and BMI of subjects of both early-onset and late-onset PIH groups at the 31st week recording was not significant compared to their own 12th week recording. The BHR of early-onset group was significantly more than the late-onset group at all the three recordings $(P=.0381$ at the 12th week, .0215 at the 24th week, and .0094 at the 31st week recordings). Though there was no difference in SBP between these two groups at the 12th week, SBP was significantly more in early-onset group compared to late-onset group at 24th $(P=.0138)$ and 31st week recordings $(P=.0350)$. The 
TABLE 1: Comparison of age, body weight (BW), body mass index (BMI), heart rate (HR), systolic blood pressure (SBP), and diastolic blood pressure (DBP) of the subjects of control group (subjects with normal pregnancy) with the subjects of study group I (those who had high risk for PIH, but did not develop PIH) and study group II (those who developed PIH later) at different weeks of pregnancy.

\begin{tabular}{|c|c|c|c|c|}
\hline $\begin{array}{l}\text { Parameters at different } \\
\text { weeks }\end{array}$ & $\begin{array}{l}\text { Control group } \\
\quad(n=38)\end{array}$ & $\begin{array}{l}\text { Study group I } \\
\quad(n=146)\end{array}$ & $\begin{array}{l}\text { Study group II } \\
\quad(n=31)\end{array}$ & $P$-values \\
\hline \multicolumn{5}{|l|}{ At 12 th week } \\
\hline Age (yr) & $24.21 \pm 3.12$ & $23.85 \pm 4.15$ & $25.30 \pm 3.60$ & .1734 \\
\hline BW $(\mathrm{kg})$ & $49.84 \pm 10.70$ & $53.10 \pm 9.70$ & $56.80 \pm 15.06^{*}$ & .0303 \\
\hline BMI $\left(\mathrm{Kg} / \mathrm{m}^{2}\right)$ & $20.26 \pm 3.98$ & $22.05 \pm 4.76$ & $24.32 \pm 8.75^{* *}$ & .0088 \\
\hline BHR (per min) & $74.44 \pm 13.77$ & $80.85 \pm 12.80^{*}$ & $90.54 \pm 15.54^{* * *, \# \#}$ & $<.0001$ \\
\hline SBP (mm Hg) & $103.60 \pm 9.32$ & $106.30 \pm 8.92$ & 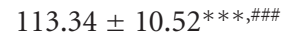 & $<.0001$ \\
\hline $\mathrm{DBP}(\mathrm{mm} \mathrm{Hg})$ & $59.34 \pm 7.78$ & $61.05 \pm 7.90$ & $65.90 \pm 7.30^{* *, \# \#}$ & .0016 \\
\hline \multicolumn{5}{|l|}{ At 24th week } \\
\hline BW (kg) & $52.81 \pm 10.81$ & $55.70 \pm 10.80$ & $60.72 \pm 14.84^{*}$ & .0171 \\
\hline $\operatorname{BMI}\left(\mathrm{Kg} / \mathrm{m}^{2}\right)$ & $21.97 \pm 4.01$ & $23.50 \pm 7.15$ & $26.56 \pm 8.20^{*}$ & .0208 \\
\hline BHR (per min) & $78.92 \pm 13.72$ & $83.60 \pm 11.70$ & $91.24 \pm 15.70^{* * *, \# \#}$ & .0004 \\
\hline SBP (mm Hg) & $104.44 \pm 7.92$ & $108.40 \pm 9.64$ & 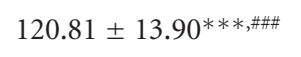 & $<.0001$ \\
\hline $\mathrm{DBP}(\mathrm{mm} \mathrm{Hg})$ & $59.89 \pm 6.14$ & $62.56 \pm 8.88$ & 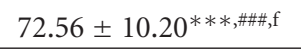 & $<.0001$ \\
\hline \multicolumn{5}{|l|}{ At 31st week } \\
\hline BW $(\mathrm{kg})$ & $56.28 \pm 10.81^{\mathrm{f}}$ & $59.10 \pm 11.84^{\mathrm{fff}, \mathrm{a}}$ & $65.36 \pm 16.40^{* *, \#}$ & .0110 \\
\hline $\operatorname{BMI}\left(\mathrm{Kg} / \mathrm{m}^{2}\right)$ & $23.89 \pm 4.00^{\mathrm{f}}$ & $26.05 \pm 5.70^{\mathrm{fff}, \mathrm{aaa}}$ & $28.80 \pm 8.50^{* *}$ & .0039 \\
\hline BHR (per min) & $87.08 \pm 14.40^{\mathrm{fff}, \mathrm{a}}$ & $91.30 \pm 10.90^{\mathrm{fff} \text {,aaa }}$ & $96.24 \pm 14.20^{* *}$ & .0096 \\
\hline SBP (mm Hg) & $104.77 \pm 8.26$ & $110.26 \pm 8.80^{* *, \mathrm{ff}}$ & $128.78 \pm 11.1^{* * *, \# \#, f f f}$ & $<.0001$ \\
\hline $\mathrm{DBP}(\mathrm{mm} \mathrm{Hg})$ & $62.00 \pm 7.79$ & $65.30 \pm 7.92^{\mathrm{fff}, \mathrm{a}}$ & 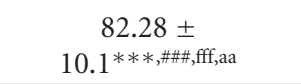 & $<.0001$ \\
\hline
\end{tabular}

The values are mean $\pm \mathrm{SD}$; analysis of each parameter between the groups and across the three weeks was done by one-way ANOVA, followed by TukeyKramer post-hoc. The ${ }^{*}$ mark indicates comparison with control group and the ${ }^{\#}$ mark indicates comparison with study group I. ${ }^{*} P<.05$; $* * P .01$; ${ }^{* * *} P<.001$; ${ }^{\#} P<.05 ;{ }^{\# \#} P<.01$; ${ }^{\# \#} P<.001$. The ${ }^{\mathrm{f}}$ indicates comparison with the 12 th week and the ${ }^{\text {a }}$ indicates comparison with the 24 th week of respective groups. ${ }^{\mathrm{f}} P<.05 ;{ }^{\mathrm{fff}} P<.001 ;{ }^{\mathrm{a}} P<.05 ;{ }^{\text {aa }} P<.01$. ( $n=29$ in study group at the 31 st week).

SBP of both the groups was significantly more at 31 st week recording compared to their own 12th week recordings, in which the significance was more for early-onset $(P<.01)$ compared to late-onset $(P<.05)$ subjects. The DBP of early-onset group was significantly more than the late-onset group at all the three weeks of recordings $(P=.0052$ at the 12th week, .0029 at the 24th week, and .0363 at the 31 st week recordings). Moreover, the DBP of both the groups was significantly more at the 31 st week recording compared to their own 12 th week recordings $(P<.001)$ and 24 th week recordings $(P<.05)$.

3.4.2. HRV Indices. Though the total power was considerably less in early-onset group compared to late-onset group at all the three trimesters of pregnancy, the difference was significant $(P=.0291)$ only at the 12th week recording (Table 4). Though the LFnu was consistently more in earlyonset group compared to late-onset group at all the three trimesters of pregnancy, the difference was not statistically significant in any of the recordings. However, HFnu was significantly less in early-onset group compared to lateonset group at second and third trimesters of pregnancy $(P=.0324$ at the 24th week and .0266 at the 31st week recordings). The LF-HF ratio was significantly more in earlyonset group compared to late-onset group at the 12th and
31 st weeks of recordings, but not at the 24th week of recording $(P=.0308$ at the 12 th week, .1063 at the 24 th week, and .0112 at the 31 st week recordings). The mean RR was significantly less in early-onset group compared to late-onset group at all the three trimesters of pregnancy $(P=.0211$ at the 12 th week, .0062 at the 24 th week, and .0242 at the 31 st week recordings). The change in SDNN was not significant between the two groups at all the three trimesters of pregnancy. The RMSSD was significantly less in early-onset group compared to late-onset group at the 12th and 31st weeks of recordings, but not at the 24th week of recording $(P=.0361$ at the 12th week, .0999 at the 24th week, and .0157 at the 31 st week recordings).

For HRV indices across the weeks within the groups, only RMSSD was significantly less in the 31 st week $(P<.05)$ in early-onset group compared to its own 12th week recording.

3.5. Correlation Analysis. The increase in LF-HF ratio in both early-onset (Table 5) and late-onset (Table 6) groups was significantly correlated with BHR and DBP at the 12th week and with all the parameters (BHR, SBP, and DBP) except $\mathrm{BMI}$ at the 24th and 31st weeks, in which the significance was maximum for BHR and DBP at all the three weeks of recordings. Moreover, the level of significance was more in early-onset compared to late-onset group for all the 
TABLE 2: Comparison of age, body weight (BW), body mass index (BMI), heart rate (HR), systolic blood pressure (SBP), and diastolic blood pressure (DBP) of the subjects of control group (subjects with normal pregnancy) with the subjects of study group I (those who had high risk for PIH, but did not develop PIH) and study group II (those who developed PIH later) at different weeks of pregnancy.

\begin{tabular}{|c|c|c|c|c|}
\hline $\begin{array}{l}\text { Parameters at different } \\
\text { weeks }\end{array}$ & $\begin{array}{l}\text { Control group } \\
\quad(n=38)\end{array}$ & $\begin{array}{l}\text { Study group I } \\
\quad(n=146)\end{array}$ & $\begin{array}{l}\text { Study group II } \\
\quad(n=31)\end{array}$ & $P$-values \\
\hline \multicolumn{5}{|l|}{ At 12th week } \\
\hline $\mathrm{TP}\left(\mathrm{ms}^{2}\right)$ & $153.80 \pm 96.09$ & $149.90 \pm 70.50$ & $105.45 \pm 75.30^{*, \#}$ & .0099 \\
\hline LFnu & $42.61 \pm 20.66$ & $46.20 \pm 21.76^{* *}$ & $57.14 \pm 24.10^{* * *, \#}$ & $<.0001$ \\
\hline HFnu & $55.64 \pm 20.90$ & $51.90 \pm 20.84$ & $42.56 \pm 21.33^{*}$ & .0295 \\
\hline $\mathrm{LF} / \mathrm{HF}$ ratio & $1.16 \pm 1.04$ & $1.21 \pm 0.94$ & $2.45 \pm 2.10^{* * *, \# \# \#}$ & $<.0001$ \\
\hline Mean RR (s) & $0.806 \pm 0.10$ & $0.75 \pm 0.11^{*}$ & $0.665 \pm 0.12^{* * *, \# \#}$ & $<.0001$ \\
\hline $\operatorname{SDNN}(\mathrm{s})$ & $0.028 \pm 0.014$ & $0.027 \pm 0.013$ & $0.020 \pm 0.013^{*, \#}$ & .0187 \\
\hline RMSSD (ms) & $30.41 \pm 20.54$ & $30.07 \pm 18.80$ & $28.46 \pm 18.80$ & .8983 \\
\hline \multicolumn{5}{|l|}{ At 24 th week } \\
\hline $\mathrm{TP}\left(\mathrm{ms}^{2}\right)$ & $149.50 \pm 69.29$ & $130.50 \pm 60.76^{\mathrm{f}}$ & 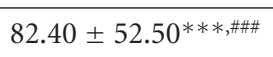 & $<.0001$ \\
\hline LFnu & $45.92 \pm 20.37$ & $50.30 \pm 20.82$ & $65.56 \pm 21.70^{* * *, \# \# \#}$ & .0003 \\
\hline HFnu & $50.07 \pm 20.37$ & $46.90 \pm 20.92$ & $40.20 \pm 21.18$ & .1383 \\
\hline LF-HF ratio & $1.38 \pm 1.04$ & $1.68 \pm 1.05^{\mathrm{ff}}$ & $2.42 \pm 1.90^{* *, \# \#}$ & .0014 \\
\hline Mean RR (s) & $0.760 \pm 0.09$ & $0.71 \pm 0.09^{*, f f}$ & $0.657 \pm 0.11^{* * *, \#}$ & $<.0001$ \\
\hline $\operatorname{SDNN}(s)$ & $0.027 \pm 0.012$ & $0.026 \pm 0.009$ & $0.022 \pm 0.009$ & .0685 \\
\hline RMSSD (ms) & $28.10 \pm 18.42$ & $26.20 \pm 15.80$ & $20.32 \pm 14.16$ & .1100 \\
\hline \multicolumn{5}{|l|}{ At 31st week } \\
\hline $\mathrm{TP}\left(\mathrm{ms}^{2}\right)$ & $143.26 \pm 86.53$ & $112.84 \pm 50.80^{*, \mathrm{fff}, \mathrm{a}}$ & $70.51 \pm 49.33^{* * *, \# \#}$ & $<.0001$ \\
\hline LFnu & $54.51 \pm 22.83^{\mathrm{f}}$ & $57.21 \pm 22.60^{\mathrm{fff}, \mathrm{a}}$ & $67.84 \pm 23.70^{*}$ & .0415 \\
\hline HFnu & $45.48 \pm 22.83$ & $41.40 \pm 20.60^{\mathrm{fff}}$ & $35.65 \pm 18.10$ & .1588 \\
\hline LF-HF ratio & $2.01 \pm 1.20^{\mathrm{ff}, \mathrm{a}}$ & $2.21 \pm 1.45^{\mathrm{fff} \text {,aаa }}$ & $3.14 \pm 2.10^{* *, \# \#}$ & .0051 \\
\hline Mean RR (s) & $0.689 \pm 0.13^{\mathrm{fff}, \mathrm{a}}$ & $0.67 \pm 0.13^{\mathrm{fff} \text {,aa }}$ & $0.624 \pm 0.11$ & .1074 \\
\hline $\operatorname{SDNN}(s)$ & $0.028 \pm 0.020$ & $0.025 \pm 0.01$ & $0.022 \pm 0.010$ & .1433 \\
\hline RMSSD (ms) & $27.29 \pm 16.79$ & $20.80 \pm 14.90^{*, \mathrm{fff}, \mathrm{a}}$ & $18.05 \pm 12.10^{*, \mathrm{f}}$ & .0240 \\
\hline
\end{tabular}

The values are mean \pm SD; analysis of each parameter between the groups and across the three weeks was done by one-way ANOVA, followed by TukeyKramer post hoc. The * mark indicates comparison with control group and the ${ }^{\#}$ mark indicates comparison with study group I. ${ }^{*} P<.05 ; * * P<.01$; ${ }^{* * *} P<.001 ;{ }^{\#} P<.05 ;{ }^{\# \#} P<.01 ;{ }^{\# \#} P<.001$. The ${ }^{\mathrm{f}}$ indicates comparison with the 12 th week and the ${ }^{\text {a }}$ indicates comparison with the 24 th week of respective groups. ${ }^{\mathrm{f}} P<.05$; ${ }^{\text {fff }} P<.001 ;{ }^{\mathrm{a}} P<.05 ;(n=29$ in study group at the 31 st week).

parameters at all the three trimesters. The correlation of LFHF ratio was not significant with any of these parameters in control group (Table 7), whereas the correlation was significant only with DBP at the 24 th and the 31 st weeks in study group I (Table 8).

\section{Discussion}

In the present study, a persistently high basal heart rate (BHR) at all the three trimesters of pregnancy in subjects of study group II who developed PIH later in pregnancy indicates that these subjects had lower vagal tone since their early part of pregnancy, as BHR is an index of parasympathetic activity [12]. The PIH subjects had significantly high systolic blood pressure (SBP) and diastolic blood pressure (DBP) in all the three trimesters of pregnancy, which could be due to their increased sympathetic tone as $\mathrm{PIH}$ is primarily a state of sympathetic overactivity $[5,6]$. SBP is the index of cardiac output that primarily depends on the stroke volume, which is determined mainly by venous return and myocardial contractility [7]. As in PIH, there is decreased blood volume, $\mathrm{PIH}$ being a state of volume depletion $[6,13]$, increased cardiac output in PIH is principally due to increased myocardial contractility, which is influenced by sympathetic activity [7]. In PIH subjects, this was further supported by considerable increase in DBP, which is an index of peripheral vascular resistance that reflects the basal sympathetic tone [7]. Thus, the findings of the present study clearly depict the presence of sympathetic activation in PIH subjects since their 1st trimester, which was further accentuated in later weeks with the advancement of pregnancy.

LF-HF ratio, the index of sympathovagal homeostasis represents the balance between sympathetic and parasympathetic activities of the individual at any given time in resting supine conditions [14]. Lesser values of this ratio indicate parasympathetic dominance and greater values indicate sympathetic dominance [14]. LF-HF ratio was significantly high in PIH subjects in comparison to the subjects of study group I and control group at all the three trimesters of recordings, reflecting increased sympathetic tone in these 
TABLE 3: Comparison of age, body weight (BW), body mass index (BMI), heart rate (HR), systolic blood pressure (SBP) and diastolic blood pressure (DBP) between early onset PIH (before completion of the 34 weeks) and late onset PIH (after completion of the 34 weeks).

\begin{tabular}{lccc}
\hline $\begin{array}{l}\text { Parameters at } \\
\text { diff. weeks }\end{array}$ & $\begin{array}{c}\text { Early onset PIH } \\
(n=15)\end{array}$ & $\begin{array}{c}\text { Late onset PIH } \\
(n=16)\end{array}$ & $P$ values \\
\hline At 12th week & & & \\
\hline Age & $24.46 \pm 4.10$ & $25.94 \pm 2.80$ & .2474 \\
BW & $61.20 \pm 14.80$ & $54.75 \pm 13.00$ & .2068 \\
BMI & $25.47 \pm 9.90$ & $23.80 \pm 6.17$ & .5746 \\
BHR & $93.96 \pm 10.22$ & $86.41 \pm 9.12$ & .0381 \\
SBP & $117.66 \pm 9.23$ & $110.41 \pm 10.68$ & .0531 \\
DBP & $67.50 \pm 6.10$ & $61.33 \pm 5.25$ & .0052 \\
\hline At 24th week & & & \\
\hline BW & $62.50 \pm 18.50$ & $58.30 \pm 13.10$ & .4693 \\
BMI & $26.97 \pm 10.20$ & $25.13 \pm 8.26$ & .5841 \\
BHR & $94.16 \pm 9.071$ & $86.58 \pm 8.30$ & .0215 \\
SBP & $125.10 \pm 8.10$ & $116.66 \pm 9.69$ & .0138 \\
DBP & $76.40 \pm 7.05$ & $67.50 \pm 8.10$ & .0029 \\
\hline At 31st week & & & \\
\hline BW & $67.83 \pm 16.76$ & $63.75 \pm 13.32$ & .4711 \\
BMI & $28.90 \pm 10.56$ & $28.52 \pm 7.50$ & .6764 \\
BHR & $100.33 \pm 10.69$ & $90.50 \pm 8.24$ & .0094 \\
SBP & $131.80 \pm 9.35^{\mathrm{ff}}$ & $122.33 \pm 12.84^{\mathrm{f}}$ & .0350 \\
DBP & $86.80 \pm 13.76^{\mathrm{ffff}, \mathrm{a}}$ & $76.24 \pm 12.04^{\mathrm{fff}, \mathrm{a}}$ & .0363 \\
\hline
\end{tabular}

The values are mean $\pm \mathrm{SD}$; Analysis of each parameter across the three weeks, one-way ANOVA, followed by Tukey-Kramer post-hoc was used. The $P$ values depicted in the last column indicate comparison between early-onset and late-onset PIH groups. The ${ }^{\mathrm{f}}$ indicates comparison with the 12th week and the ${ }^{a}$ indicates comparison with the 24th week of respective groups. ${ }^{\mathrm{f}} P<.05$; ${ }^{\text {ff }} P<.01$; ${ }^{\text {fff }} P<.001 ;{ }^{\text {a }} P<.05 ;(n=13$ in early-onset group at the 31 st week).

subjects. This was further evidenced by significant increase in LFnu in PIH subjects in comparison to other two groups at all the three gestational weeks as LFnu mainly reflects sympathetic modulation of heart functions [14]. However, sympathovagal imbalance in PIH was also contributed by vagal withdrawal, at least in the early part of pregnancy, as HFnu represents vagal modulation of SA nodal discharge [14], which was decreased in PIH group compared to the control group at the 12th week of recording. Further, significant decrease in total power (TP) in PIH subjects compared to control subjects at all the three trimesters establishes the contribution of vagal withdrawal in the development of sympathovagal imbalance, as TP in general represents the parasympathetic potency of cardiac modulation [11]. Though few earlier reports had indicated some kind of prediction of PIH in early part pregnancy through serial assessment of cardiovascular control [15], heart rate and blood pressure variabilities [16], heart rate and blood velocity variability and uterine perfusion combined with heart rate and blood pressure variabilities [17], none of these studies had analyzed the nature and degree of sympathovagal
TABLE 4: Comparison of HRV indices between early-onset PIH and late-onset PIH at different weeks of gestation.

\begin{tabular}{|c|c|c|c|}
\hline $\begin{array}{l}\text { HRV indices sat } \\
\text { diff. weeks }\end{array}$ & $\begin{array}{l}\text { Early onset PIH } \\
\quad(n=15)\end{array}$ & $\begin{array}{l}\text { Late onset PIH } \\
\quad(n=16)\end{array}$ & $P$ values \\
\hline \multicolumn{4}{|c|}{ At 12th week } \\
\hline $\mathrm{TP}\left(\mathrm{ms}^{2}\right)$ & $75.80 \pm 61.20$ & $135.90 \pm 82.20$ & .0291 \\
\hline LFnu & $63.42 \pm 25.30$ & $52.40 \pm 20.85$ & .1948 \\
\hline HFnu & $34.50 \pm 16.50$ & $47.90 \pm 20.50$ & .0554 \\
\hline LF-HF ratio & $2.96 \pm 1.70$ & $1.80 \pm 1.10$ & .0308 \\
\hline Mean RR (s) & $0.61 \pm 0.13$ & $0.71 \pm 0.097$ & .0211 \\
\hline $\operatorname{SDNN}(\mathrm{s})$ & $0.020 \pm 0.014$ & $0.021 \pm 0.019$ & .8694 \\
\hline RMSSD (ms) & $21.95 \pm 13.10$ & $36.41 \pm 20.26$ & .0263 \\
\hline \multicolumn{4}{|l|}{ At 24 th week } \\
\hline $\mathrm{TP}\left(\mathrm{ms}^{2}\right)$ & $70.80 \pm 60.50$ & $118.40 \pm 75.20$ & .0630 \\
\hline LFnu & $67.06 \pm 30.08$ & $54.08 \pm 24.41$ & .1961 \\
\hline HFnu & $30.75 \pm 17.34$ & $45.95 \pm 20.10$ & .0324 \\
\hline LF-HF ratio & $3.05 \pm 1.80$ & $2.02 \pm 1.64$ & .1063 \\
\hline Mean RR (s) & $0.59 \pm 0.122$ & $0.70 \pm 0.083$ & .0062 \\
\hline $\operatorname{SDNN}(\mathrm{s})$ & $0.022 \pm 0.011$ & $0.022 \pm 0.005$ & .9999 \\
\hline RMSSD (ms) & $17.75 \pm 8.01$ & $23.96 \pm 15.22$ & .1700 \\
\hline \multicolumn{4}{|l|}{ At 31st week } \\
\hline $\mathrm{TP}\left(\mathrm{ms}^{2}\right)$ & $58.30 \pm 42.65$ & $98.70 \pm 69.10$ & .0769 \\
\hline LFnu & $74.46 \pm 32.83$ & $60.05 \pm 28.15$ & .2139 \\
\hline HFnu & $24.40 \pm 15.60$ & $39.80 \pm 19.02$ & .0266 \\
\hline LF-HF ratio & $3.84 \pm 1.50$ & $2.50 \pm 1.15$ & .0112 \\
\hline Mean RR (s) & $0.58 \pm 0.11$ & $0.67 \pm 0.093$ & .0242 \\
\hline SDNN (s) & $0.021 \pm 0.016$ & $0.023 \pm 0.004$ & .6316 \\
\hline RMSSD (ms) & $12.65 \pm 6.20^{\mathrm{f}}$ & $22.86 \pm 13.10$ & .0157 \\
\hline
\end{tabular}

The values are mean $\pm \mathrm{SD}$; The $P$-values depicted in the last column indicate comparison between early-onset and late-onset PIH groups. The ${ }^{f}$ indicates comparison with the 12th week value of RMSSD in early-onset group. ${ }^{\mathrm{f}} P<$ .05 ; $(n=13$ in early-onset group at the 31 st week).

TABLE 5: Correlation of LF-HF ratio with body mass index (BMI), basal heart rate (HR), systolic blood pressure (SBP), and diastolic blood pressure (DBP) of subjects of early-onset PIH group at different weeks of gestation.

\begin{tabular}{lcccccc}
\hline & \multicolumn{2}{c}{ 12th week } & \multicolumn{2}{c}{ 24th week } & \multicolumn{2}{c}{31 st week } \\
& $R$ & $P$ & $r$ & $P$ & $r$ & $P$ \\
\hline BMI & 0.092 & .153 & 0.135 & .116 & 0.312 & .085 \\
BHR & 0.770 & .000 & 0.782 & .000 & 0.809 & .000 \\
SBP & 0.360 & .052 & 0.374 & .030 & 0.426 & .018 \\
DBP & 0.564 & .003 & 0.618 & .001 & 0.694 & .000 \\
\hline
\end{tabular}

The $P$-values less than .05 were considered significant.

imbalance in different trimesters of pregnancy to elucidate the progression and prediction of PIH.

Time domain indices such as mean-RR, SDNN, and RMSSD are mainly the indicators of parasympathetic activity [11]. In the present study, significant decrease in mean-RR in PIH subjects at the 12th and the 24th weeks recording indicates decreased parasympathetic tone in these subjects in the first half of pregnancy. SDNN and RMSSD reflect vagal 
TABLE 6: Correlation of LF-HF ratio with body mass index (BMI), basal heart rate (HR), systolic blood pressure (SBP), and diastolic blood pressure (DBP) of subjects of late-onset $\mathrm{PIH}$ group at different weeks of gestation.

\begin{tabular}{lcccccc}
\hline & \multicolumn{2}{c}{ 12th week } & \multicolumn{2}{c}{ 24th week } & \multicolumn{2}{c}{31 st week } \\
& $R$ & $P$ & $r$ & $P$ & $r$ & $P$ \\
\hline BMI & 0.020 & .205 & 0.054 & .170 & 0.011 & .110 \\
BHR & 0.570 & .003 & 0.632 & .001 & 0.710 & .000 \\
SBP & 0.230 & .090 & 0.284 & .075 & 0.385 & .046 \\
DBP & 0.448 & .032 & 0.510 & .010 & 0.610 & .001 \\
\hline
\end{tabular}

The $P$-values less than .05 were considered significant.

TABLE 7: Correlation of LF-HF ratio with body mass index (BMI), basal heart rate (HR), systolic blood pressure (SBP), and diastolic blood pressure (DBP) of subjects of control group at different weeks of gestation.

\begin{tabular}{lcccccc}
\hline & \multicolumn{2}{c}{ 12th week } & \multicolumn{2}{c}{ 24th week } & \multicolumn{2}{c}{ 31st week } \\
& $R$ & $P$ & $r$ & $P$ & $r$ & $P$ \\
\hline BMI & 0.034 & .245 & 0.020 & .310 & 0.010 & .465 \\
BHR & 0.204 & .102 & 0.210 & .095 & 0.240 & .086 \\
SBP & 0.130 & .170 & 0.184 & .135 & 0.232 & .090 \\
DBP & 0.118 & .190 & 0.125 & .172 & 0.184 & .125 \\
\hline
\end{tabular}

The $P$-values less than .05 were considered significant.

TABLE 8: Correlation of LF-HF ratio with body mass index (BMI), basal heart rate (HR), systolic blood pressure (SBP), and diastolic blood pressure (DBP) of subjects of study group I at different weeks of gestation.

\begin{tabular}{lcccccc}
\hline & \multicolumn{2}{c}{ 12th week } & \multicolumn{2}{c}{ 24th week } & \multicolumn{2}{c}{31 st week } \\
& $r$ & $P$ & $r$ & $P$ & $r$ & $P$ \\
\hline BMI & 0.031 & .260 & 0.048 & .235 & 0.018 & .380 \\
BHR & 0.189 & .132 & 0.218 & .105 & 0.231 & .092 \\
SBP & 0.096 & .124 & 0.235 & .086 & 0.280 & .065 \\
DBP & 0.290 & .071 & 0.312 & .048 & 0.346 & .020 \\
\hline
\end{tabular}

The $P$-values less than .05 were considered significant.

modulation of heart rate, and RMSSD is considered as an important indicator of parasympathetic influence especially in long-term HRV recordings [11]. Though HRV recording in the present study is a short-term one, the significant decrease in RMSSD at the 31st week in study group II indicates mild decrease in parasympathetic modulation of SA nodal discharge towards later part of pregnancy in $\mathrm{PIH}$ subjects.

Till date, to best of our knowledge, the cause of sympathovagal imbalance in PIH has not been elucidated. One could argue that higher BMI in PIH subjects has contributed to the autonomic imbalance as increased BMI has been reported to increase sympathetic activity [18]. However, increase in body weight and BMI in pregnancy is mainly due to increase in size of the growing fetus, uterus, and placenta, and increase in ECF volume, not due to increased adiposity [19]. Moreover, recent report from our laboratory indicates that there is no correlation of BMI with sympathovagal imbalance in PIH [9]. From the present study, it appears that in normal pregnancy from first trimester to third trimester, there is progressive increase in sympathetic activity without much decrease in parasympathetic activity as LFnu but not HFnu was altered significantly at the 31st week in control subjects compared to their 12 th week recording. Hence, PIH was proposed to be a state of exaggeration of this increased sympathetic activity of the normal pregnancy [5].

In the present study, there was no significant difference in age, body weight, and BMI between subjects of earlyonset and late-onset PIH. Significantly high BHR, SBP (except in the 12th week) and DBP in early-onset group compared to the late-onset group at all three weeks of gestation indicate higher degree sympathetic activity in these subjects throughout pregnancy. This was confirmed by presence of significantly high LF-HF ratio in early-onset group compared to late-onset group in the 12th and the 31 st weeks of pregnancy. Mean RR was significantly low in early onset group in all the three trimesters as heart rate was significantly high in these subjects. Significantly low RMSSD in early-onset group at the 12th and the 31 st week indicates vagal inhibition in these subjects, as RMSSD is a marker of vagal modulation of heart rate [11]. It appears that contribution of vagal withdrawal is more prominent than sympathetic inhibition in the genesis of sympathovagal imbalance in early-onset $\mathrm{PIH}$, as decrease in HFnu in early-onset subjects compared to late-onset subjects was significant at all the three trimesters, without significant increase in LFnu. This was further indicated by a proportionate correlation of LH-FH ratio with increase in BHR across the three trimesters of gestation in both early-onset and late-onset groups, as BHR is an index of vagal activity [12]. This correlation was more in early-onset group indicating a greater vagal withdrawal in these subjects compared to late-onset subjects. The degree of correlation of LF-HF ratio with SBP and DBP was less significant in comparison to the degree of correlation with BHR indicating that the impact of sympathetic overactivity is relatively less in comparison to vagal inhibition. Contribution of BMI to the sympathovagal imbalance in both early-onset and late-onset groups is negligible, as the correlation was not significant for this parameter.

Based on the report of our hospital records and others, the patients with early-onset PIH more commonly enter the phase of eclampsia that heralds convulsion and other complications. Therefore, in our study we had kept all our subjects those who had LF-HF ratio more than 2.5 at their 31 st week recording under constant medical surveillance till their delivery. Hence, none of our subject had progressed into the eclamptic phase. Thus, it is imperative to predict the onset of PIH in subjects having risks for PIH, especially those who are prone for early-onset type of this dysfunction. However, the future research should aim at elucidating the mechanisms that contribute to vagal withdrawal in earlyonset PIH subjects.

Though the exact cause of sympathovagal imbalance in PIH is not known, recently, it was suggested that hypertension in pregnancy is due to release of biologically active circulating factors such as cytokines or reactive oxygen 
species from placenta (placental factors) that inhibit vascular relaxation pathway or promote vascular smooth muscle contraction [20]. As present study indicates sympathovagal imbalance mainly in the form of vagal withdrawal in earlyonset PIH, we hypothesize that the so-called placental factors might be acting primarily on medullary cardiovascular centers in the brain to modulate central parasympathetic outflow rather than exclusively acting peripherally on the blood vessels to alter vascular tone, as reports indicate that cytokines [21, 22] and reactive oxygen species [22, 23] released peripherally cross blood-brain barrier and influence activities of various brain centers. Also, it appears that sympathetic overactivity in $\mathrm{PIH}$ is not just exaggeration of sympathetic activation that usually occurs towards later part of normal pregnancy [24], it is an abnormal autonomic reaction that starts in the first trimester with formation of placenta. Therefore, further studies should address the nonpharmacological intervention such as breathing exercises program that are known to promote vagal tone and decrease sympathetic activity [25], in the early part of pregnancy in women with risk factor for PIH having significant sympathovagal imbalance, so that they do not progress into the phase of hypertension.

\section{Limitations of the Study}

The major limitation of the present study is less sample size of early-onset and late-onset PIH groups. Also, we have not studied the role of cytokines released from placenta such as interleukins in the genesis of sympathovagal imbalance. In our future studies, we plan to assess the biochemical markers of sympathovagal imbalance in PIH in a lager sample size. The sympathetic activity measured in this study is not accurate. Therefore, future studies should include more accurate methods of assessment of sympathetic activity such as estimation of plasma catecholamines or metabolites of catecholamines in urine like vanillylmandelic acid (VMA), metanephrine, and normetanephrine.

\section{Conflict of Interests}

The authors declare that there is no conflict of interests.

\section{References}

[1] J. P. Greenwood, J. B. Stoker, J. J. Walker, and D. A. S. G. Mary, "Sympathetic nerve discharge in normal pregnancy and pregnancy-induced hypertension," Journal of Hypertension, vol. 16, no. 5, pp. 617-624, 1998.

[2] P. Gupta and O. P. Ghai, "Common complications of pregnancy," in Preventive and Social Medicine, pp. 358-361, CBS Publications, New Delhi, India, 2nd edition, 1998.

[3] S. J. Wagner, S. Barac, and V. D. Garovic, "Hypertensive pregnancy disorders: current concepts," Journal of Clinical Hypertension, vol. 9, no. 7, pp. 560-566, 2007.

[4] M. Joydev, L. S. Subroto, and B. B. Geeta, "Current concepts in PIH," in Current Obstetrics and Gynecology, G. M. Geeta, Ed., pp. 93-100, Jaypee Publications, New Delhi, India, 1st edition, 2007.
[5] H. P. Schobel, T. Fischer, K. Heuszer, H. Geiger, and R. E. Schmieder, "Preeclampsia: a state of sympathetic overactivity," The New England Journal of Medicine, vol. 335, no. 20, pp. 1480-1485, 1996.

[6] W. Visser and H. C. S. Wallenburg, "Central hemodynamic observations in untreated preeclamptic patients," Hypertension, vol. 17, no. 6, pp. 1072-1077, 1991.

[7] W. F. Ganong, "Cardiovascular regulatory mechanisms," in Review of Medical Physiology, K. E. Barrett, S. Boitano, S. M. Barman, and H. L. Brooks, Eds., pp. 555-568, Tata McGraw Hill Education Private Limited, New Delhi, India, 23rd edition, 2010.

[8] G. K. Pal, P. Shyma, S. Habeebullah, P. Shyjus, and P. $\mathrm{Pal}$, "Spectral analysis of heart rate variability for early prediction of pregnancy-induced hypertension," Clinical and Experimental Hypertension, vol. 31, no. 4, pp. 330-341, 2009.

[9] P. Shyma, G. K. Pal, S. Habeebullah, P. Shyjus, and P. Pal, "Decreased total power of HRV with increased LF power in early part of pregnancy predicts development PIH in Indian population," Biomedicine, vol. 28, no. 2, pp. 104-107, 2008.

[10] K. Duckitt and D. Harrington, "Risk factors for pre-eclampsia at antenatal booking: systematic review of controlled studies," British Medical Journal, vol. 330, no. 7491, pp. 565-567, 2005.

[11] "Heart rate variability: standards of measurement, physiological interpretation and clinical use. Task Force of the European Society of Cardiology and the North American Society of Pacing and Electrophysiology," Circulation, vol. 93, no. 5, pp. 1043-1065, 1996.

[12] C. J. Mathias and R. Bannister, "Investigation of autonomic disorders," in Autonomic Failure: A Textbook of Clinical Disorders of Autonomic Nervous System, R. S. Bannister and C. J. Mathias, Eds., pp. 255-290, Oxford University Press, Oxford, UK, 3rd edition, 1992.

[13] D. A. Courtar, M. E. A. Spaanderman, R. Aardenburg, B. J. A. Janssen, and L. L. H. Peeters, "Low plasma volume coincides with sympathetic hyperactivity and reduced baroreflex sensitivity in formerly preeclamptic patients," Journal of the Society for Gynecologic Investigation, vol. 13, no. 1, pp. 48-52, 2006.

[14] A. Malliani, "Heart rate variability: from bench to bedside," European Journal of Internal Medicine, vol. 16, no. 1, pp. 1220, 2005.

[15] S. Rang, H. Wolf, G. A. V. Montfrans, and J. M. Karemaker, "Serial assessment of cardiovascular control shows early signs of developing pre-eclampsia," Journal of Hypertension, vol. 22, no. 2, pp. 369-376, 2004.

[16] E. M. K. Ekholm, K. U. O. Tahvanainen, and T. Metsälä, "Heart rate and blood pressure variabilities are increased in pregnancy-induced hypertension," American Journal of Obstetrics and Gynecology, vol. 177, no. 5, pp. 1208-1212, 1997.

[17] T. Walther, N. Wessel, H. Malberg, A. Voss, H. Stepan, and R. Faber, "A combined technique for predicting preeclampsia: concurrent measurement of uterine perfusion and analysis of heart rate and blood pressure variability," Journal of Hypertension, vol. 24, no. 4, pp. 747-750, 2006.

[18] N. E. Straznicky, G. W. Lambert, and E. A. Lambert, "Neuroadrenergic dysfunction in obesity: an overview of the effects of weight loss," Current Opinion in Lipidology, vol. 21, no. 1, pp. 21-30, 2010.

[19] F. G. Cunningham, J. L. Kenneth, L. B. Steven, C. H. John, C. G. Larry, and D. W. Katherine, "Maternal physiology," in Williams Obstetrics, chapter 5, McGraw-Hill, New York, NY, USA, 22nd edition, 2005. 
[20] A. K. Stennett and R. A. Khalil, "Neurovascular mechanisms of hypertension in pregnancy," Current Neurovascular Research, vol. 3, no. 2, pp. 131-148, 2006.

[21] J. D. Johnson, V. Cortez, S. L. Kennedy, T. E. Foley, H. Hanson III, and M. Fleshner, "Role of central $\beta$-adrenergic receptors in regulating proinflammatory cytokine responses to a peripheral bacterial challenge," Brain, Behavior, and Immunity, vol. 22, no. 7, pp. 1078-1086, 2008.

[22] J. L. Lynch and W. A. Banks, "Opiate modulation of IL- $1 \alpha$, IL2 , and TNF- $\alpha$ transport across the blood-brain barrier," Brain, Behavior, and Immunity, vol. 22, no. 7, pp. 1096-1102, 2008.

[23] R. Poulet, M. T. Gentile, C. Vecchione et al., "Acute hypertension induces oxidative stress in brain tissues," Journal of Cerebral Blood Flow and Metabolism, vol. 26, no. 2, pp. 253262, 2006.

[24] S. Rang, H. Wolf, G. A. V. Montfrans, and J. M. Karemaker, "Non-invasive assessment of autonomic cardiovascular control in normal human pregnancy and pregnancy-associated hypertensive disorders: a review," Journal of Hypertension, vol. 20, no. 11, pp. 2111-2119, 2002.

[25] T. Pramanik, H. O. Sharma, S. Mishra, A. Mishra, R. Prajapati, and S. Singh, "Immediate effect of slow pace bhastrika pranayama on blood pressure and heart rate," Journal of Alternative and Complementary Medicine, vol. 15, no. 3, pp. 293-295, 2009. 


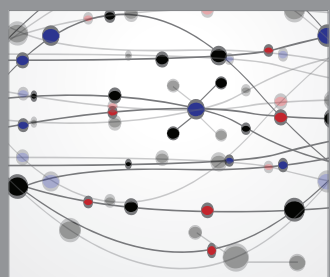

The Scientific World Journal
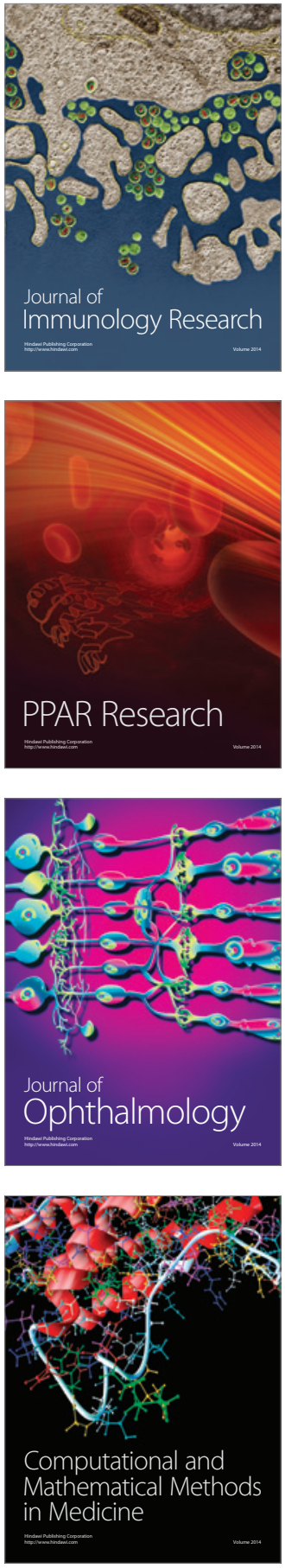

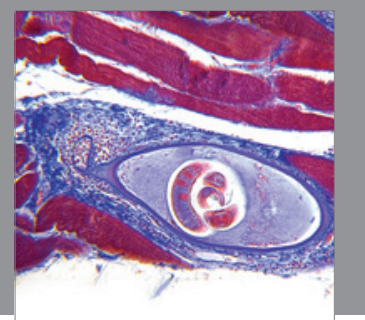

Gastroenterology

Research and Practice
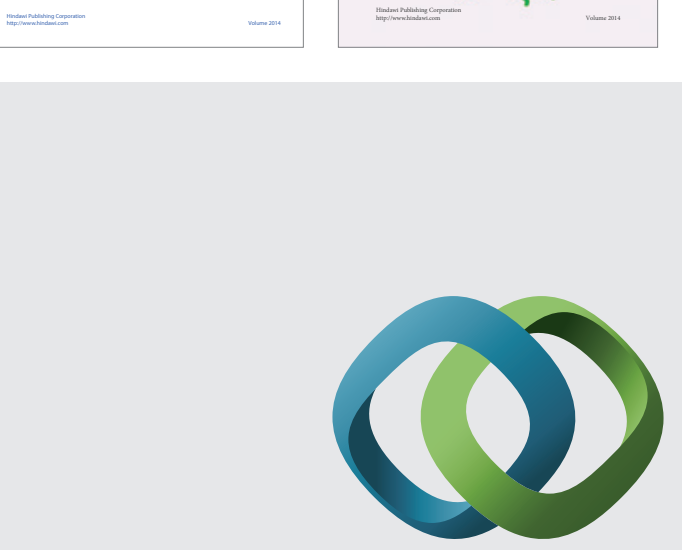

\section{Hindawi}

Submit your manuscripts at

http://www.hindawi.com
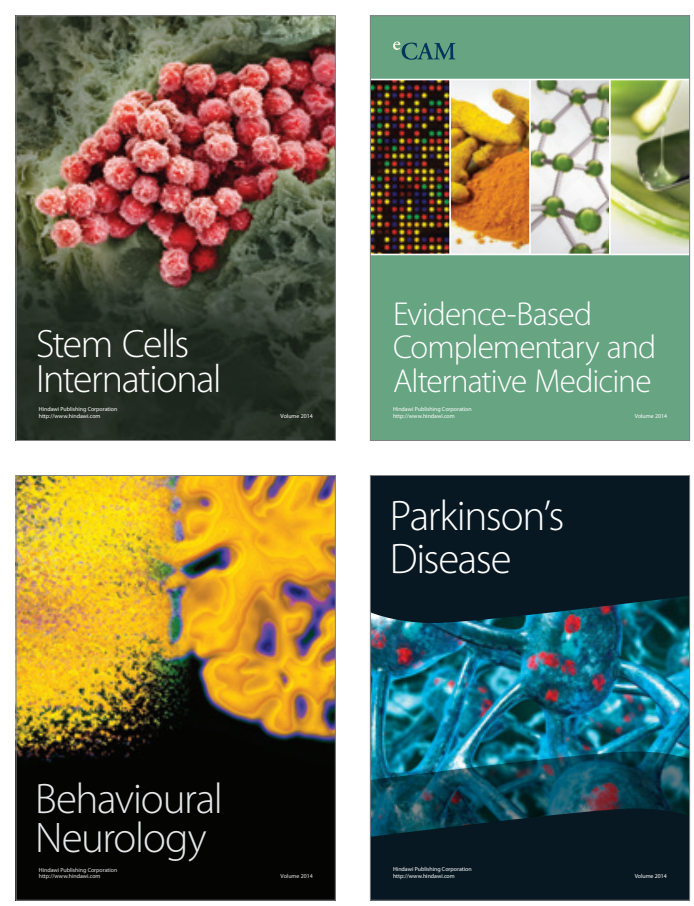

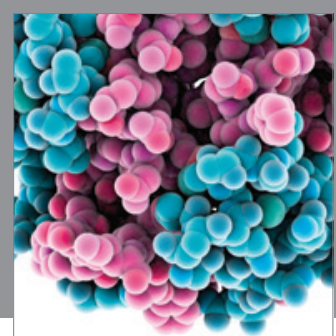

Journal of
Diabetes Research

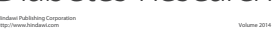

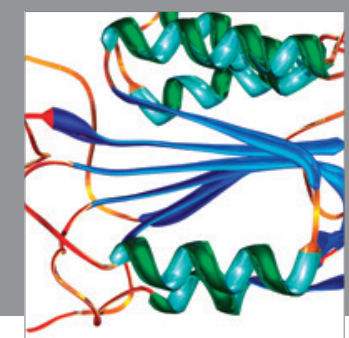

Disease Markers
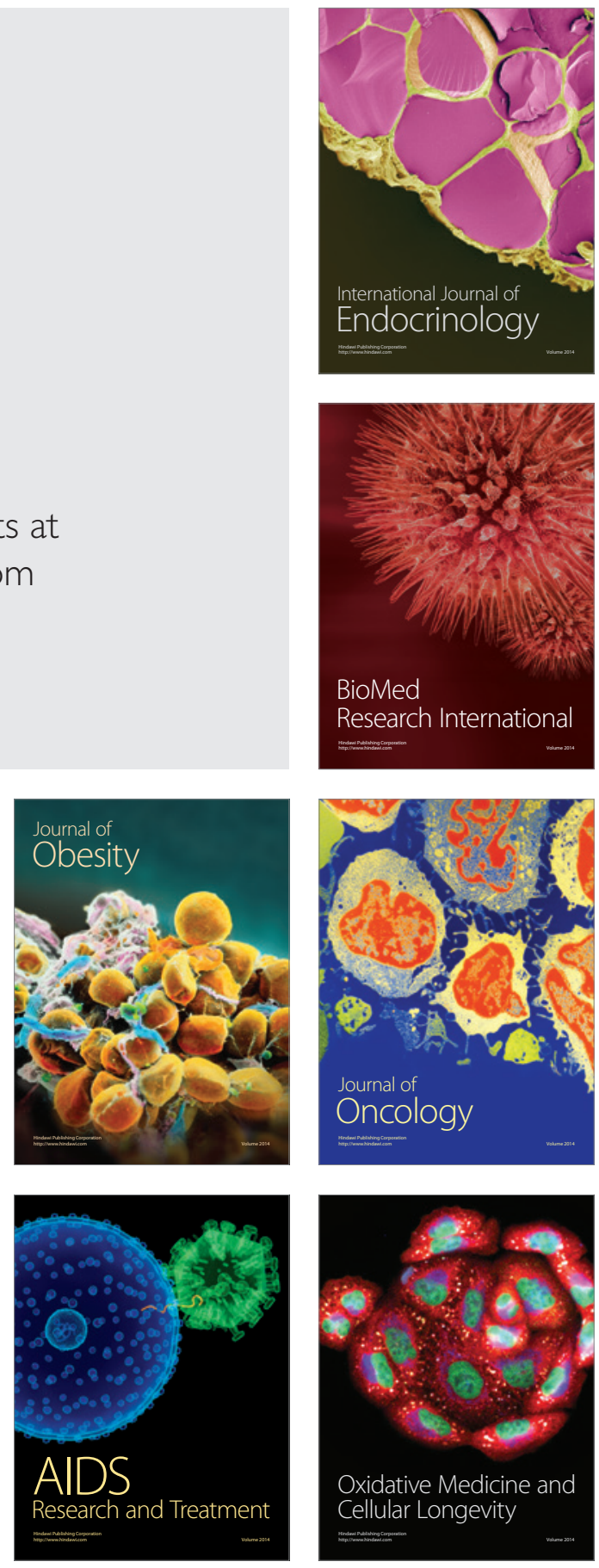